\title{
Dynamic control of liquid-core/liquid-cladding optical waveguides
}

\author{
Daniel B. Wolfe*, Richard S. Conroy ${ }^{\dagger}$, Piotr Garstecki*, Brian T. Mayers*, Michael A. Fischbach*, Kateri E. Paul*, \\ Mara Prentiss $^{\dagger}$, and George M. Whitesides*‡
}

*Department of Chemistry and Chemical Biology, Harvard University, 12 Oxford Street, Cambridge, MA 02138 ; and ${ }^{\dagger}$ Department of Physics, Harvard
University, 17 Oxford Street, Cambridge, MA 02138

Contributed by George M. Whitesides, June 22, 2004

This report describes the manipulation of light in waveguides that comprise a liquid core and a liquid cladding (liq/liq waveguide). These waveguides are dynamic: Their structure and function depend on a continuous, laminar flow of the core and cladding liquids. Because they are dynamic, they can be reconfigured and adapted continuously in ways that are not possible with solid-state waveguides. The liquids are introduced into the channels of a microfluidic network designed to sandwich the flowing core liquid between flowing slabs of the cladding fluid. At low and moderate Reynolds numbers, flow is laminar, and the liq/liq interfaces are optically smooth. Small irregularities in the solid walls of the channels do not propagate into these interfaces, and liq/liq waveguides therefore exhibit low optical loss because of scattering. Manipulating the rate of flow and the composition of the liquids tunes the characteristics of these optical systems.

$\mathbf{T}$ his report describes the design and operation of a waveguide, an optical switch, and an evanescent coupler in which both the light-guiding and cladding structures are liquids flowing at low Reynolds number $(\mathrm{Re} \approx 5-500)$ (1) in microchannels fabricated in poly(dimethylsiloxane) (PDMS). The liquid/liquid interfaces in these systems [liquid-core/liquid-cladding (liq/liq) waveguides] are optically smooth at low Re flow; modest roughness in the PDMS channel walls has little effect on the quality of this interface. We estimate that the optical loss because of scattering at the liq/liq interface is $<1 \mathrm{~dB} / \mathrm{cm}, \lambda=400-1,100$ $\mathrm{nm}$; this estimate is similar to losses observed in polymer and inorganic planar optical waveguides (2). Because the dimensions of the waveguiding region (i.e., the liquid core) in the flowing liquid streams are smaller than the dimensions of the channel and because the roughness of the edges of the channel does not degrade performance, liq/liq waveguides can be fabricated easily and rapidly in organic polymers by using the convenient techniques of rapid prototyping (3-6). Here we demonstrate the operation of liq/liq waveguides and show that their properties can be changed by manipulating the rate of flow and/or the composition of the liquids they comprise. We believe that these systems provide the basis for a tunable class of optical devices.

We (7) and others (8-15) have prepared and characterized liquid-core/solid-cladding waveguides in solid [glass $(8,12,13$, $15)$ or polymeric $(9-11,14)]$ microchannels. Liquid-core waveguiding is commonly used to increase the length of the optical path (and thus the sensitivity) of chip-based spectrophotometers $(15,16)$. Solid-core/liquid-cladding waveguides have been prepared by pumping fluid through air holes within the cladding layer of optical fibers (17-19) and by integration of bare fiber cores with liquids contained in microfluidic channels $(20,21)$. Although all of these configurations are useful, they allow reconfiguration of the optical properties of the system (e.g., the contrast in refractive index between core and cladding, the geometry of the channels, and the pattern of optical characteristics of core and cladding) only in limited ways. Liq/liq waveguides have been fabricated by using the flow of liquids from concentric glass capillaries into a single, large glass capillary (22). This method is not applicable to complex fluidic systems because it is difficult to change the geometry of the capillary and to form two or more liq/liq waveguides simultaneously.

We believe that liq/liq waveguides in PDMS microchannels have five useful properties. (i) The optical (e.g., refractive index, absorption, and fluorescence) and physical properties (e.g., magnetic susceptibility and electrical conductivity) of both of the core and the cladding of the waveguides can be changed readily, continuously, and independently by introducing different fluids. (ii) Dopants used to control optical properties (e.g., laser and fluorescent dyes) that degrade by photobleaching can be replaced continuously in use or used to create transitory or moving optical structures. (iii) The size of the liquid core can be changed dynamically to allow switching between single-mode and multimode waveguiding. (iv) The path of the liq/liq waveguide can be switched without electrical or thermal input by manipulating the rate of flow of the several independent liquid streams. (v) The dimensions and optical properties of the cladding can be changed to couple two or more cores optically; this ability is the basis for a fluidic evanescent coupler. To our knowledge, these properties provide a unique level of tunability of liq/liq optical waveguides.

\section{Materials and Methods}

We used deionized water $\left(n_{\mathrm{d}}=1.335\right)$ as the cladding and an aqueous solution of $\mathrm{CaCl}_{2}\left(5 \mathrm{M}, n_{\mathrm{d}}=1.445\right)$ as the core of the liq/liq waveguide for three reasons. First, water does not swell PDMS (23) and thus does not affect the mechanical properties of the PDMS or the dimensions of the microfluidic channels. Second, the refractive index of the core fluid is greater than $\operatorname{PDMS}\left(n_{\mathrm{d}}=1.40\right)$, and thus light will not escape from the liquid core if it comes close to the walls of the channel. Third, the interface between the core and cladding fluids is smooth at low rates of flow.

We fabricated the required microchannel systems in PDMS by using standard procedures (Fig. $1 A-C$ shows a representative design) (6). The height of all of the channels used in these experiments was $\approx 100 \mu \mathrm{m}$. The channels were designed to allow light from an optical fiber to couple easily into and out of the liq/liq waveguides. The light from an optical fiber was endcoupled into the guiding liquid before the cladding liquids were introduced. The refractive index of PDMS is less than that of the $\mathrm{CaCl}_{2}$ solution (core fluid), and thus the PDMS acts as a cladding layer for the liquid waveguide in this region of the microfluidic channel (see the supporting information, which is published on the PNAS web site). The guided light exits the liq/liq waveguide when the core fluid is forced to turn by $90^{\circ}$ with a radius of $\approx 0.5$ $\mathrm{mm}$ (much less than the critical radius) (24). We imaged the light exiting the waveguide through an optically transparent window by using a microscope objective and a charge-coupled device

Abbreviations: PDMS, poly(dimethylsiloxane); liq/liq, liquid-core/liquid-cladding; IG, illuminated waveguide; CG, coupled waveguide.

¥To whom correspondence should be addressed. E-mail: gwhitesides@gmwgroup. harvard.edu.

๑ 2004 by The National Academy of Sciences of the USA 

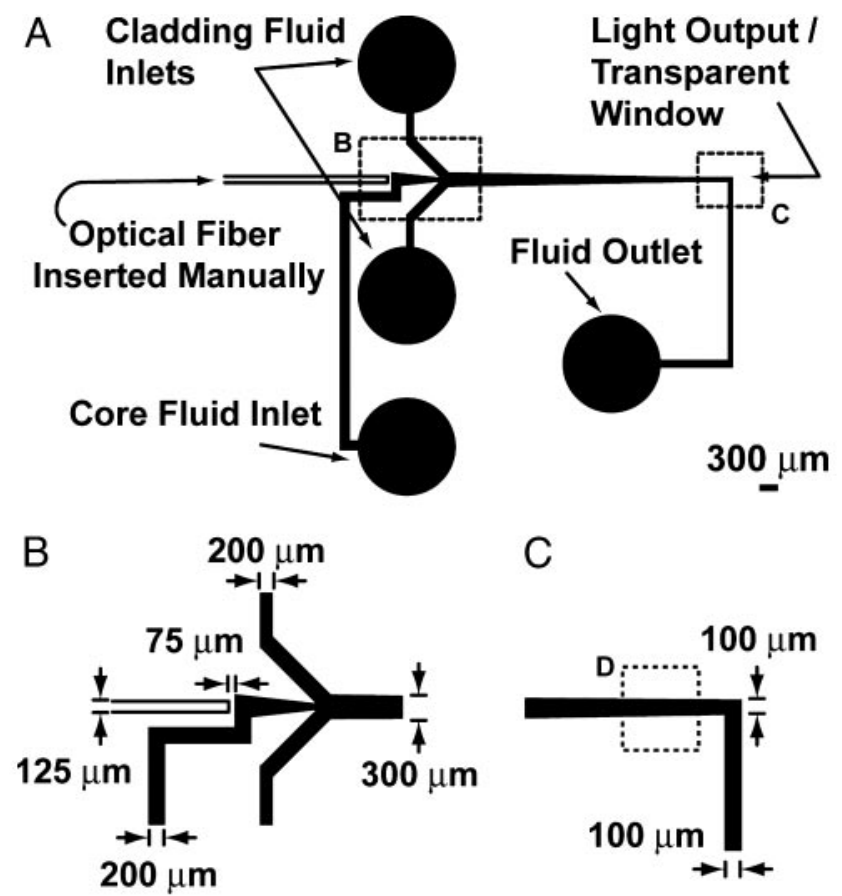

Inlet Side

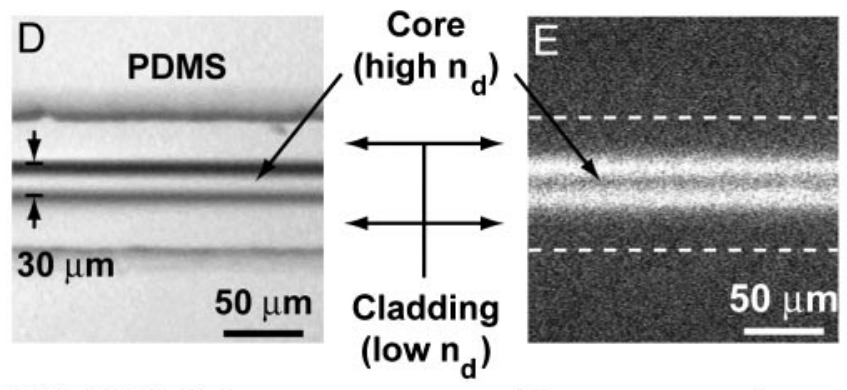

Bright Field Image

Fluorescence Image

Fig. 1. Liq/liq waveguide systems. (A) Diagram of the design of a microfluidic channel used in these experiments. $(B-C)$ Detailed diagrams of the regions of the microfluidic channel in $A$ highlighted by dashed boxes. (D) Optical micrograph of the liq/liq waveguide in the region in the dashed box in $C$. The core fluid has been dyed for visualization purposes only. $(E)$ Fluorescence micrograph of the same region of the channel as in $D$. The visible fluorescence signal has been produced by excitation with a broadband deuterium, fiber-coupled light source leaking into the evanescent field from the core of the waveguide. The dotted lines indicate the location of the walls of the microchannel.

camera. We also designed channels that have an additional inlet for an optical fiber at the end of the channel, and used this design to couple light from the liq/liq waveguide into an optical fiber. The efficiency of coupling light into a multimode optical fiber (step-index fiber, numerical aperture $=0.22, d_{\text {core }}=105 \mu \mathrm{m}$, $\left.d_{\text {outer }}=125 \mu \mathrm{m}\right)$ from the liq/liq waveguide was $\approx 40 \%$. The coupling efficiency was measured for multimode light coupled into multimode fibers. We did not characterize the efficiency of coupling single-mode light into a single-mode fiber. The light coming out of the liq/liq waveguide remains polarized in the input direction to $\approx 100: 1$; this ratio is indistinguishable from the light in the input fiber (see the supporting information).

The walls of the PDMS microfluidic channel were relatively rough (i.e., there was obvious roughness with dimensions $>5$ $\mu \mathrm{m}$; Fig. $1 D$ ). The roughness of the walls changes the shape of the liquid-liquid interfaces only by changing the cross- sectional area of the channel. When the roughness is $<5 \%$ of the total width of the channel, its effect is negligible on the interfaces between the core and cladding fluids. We doped the cladding fluid with FITC-dextran $(1 \mathrm{mg} / \mathrm{ml})$ to image the evanescent field by fluorescence microscopy (Fig. $1 E$ ); in this experiment, the light in the evanescent field excites the fluorophore contained in the cladding solution. The interface, as viewed in this image, is smooth. It is also diffuse. Three factors contribute to this lack of definition. (i) The intensity of the light emitted, and thus the signal-to-noise ratio, was low; these conditions resulted in a low-resolution image. (ii) The interface between the liquids was broadened by diffusion of $\mathrm{Ca}^{2+}$ and $\mathrm{Cl}^{-}$between the core and the cladding. (iii) The topography of this interface was not vertical to the floor of the channel but was curved (25). These characteristics prevent the quantitative interpretation of the thickness and structure of the interface between core and cladding (without extensive work by confocal microscopy) (25).

\section{Results}

Single-Mode and Multimode Waveguiding. We demonstrated the ability to switch the waveguiding from single-mode to multimode by adjusting the contrast in refractive index between core and cladding and the dimensions of the core. The refractive index contrast ( $n_{\mathrm{d} \text { core }}-n_{\mathrm{d} \text { cladding }}$ ) between the core and the cladding fluids decreases along the length of the channel because of diffusive broadening of the interface between the core into the cladding. We modeled this behavior for a liquid core of $10-\mu \mathrm{m}$ width and identified rates of flow for the core $\{2.5 \mu \mathrm{l} / \mathrm{min}$; residence time in the channel [the volume of the fluid in the channel $\left(\mathrm{cm}^{3}\right) /$ the rate of flow $\left.\left.\left(\mathrm{cm}^{3} / \mathrm{s}\right)\right], \tau=0.12 \mathrm{~s}\right\}$ and cladding $(10 \mu \mathrm{l} / \mathrm{min}, \tau=0.135 \mathrm{~s})$ fluids that yielded a contrast in refractive index at the beginning of the channel of $\approx 0.1$, but $<0.002$ (over this width) near the end of the channel (Fig. $2 A$ ); see supporting information for these data. For this contrast, single-mode guiding for light $\lambda=780 \mathrm{~nm}$ is possible only for cores with a diameter of $<10$ $\mu \mathrm{m}$ (26). We have demonstrated a core diameter of $\approx 8 \mu \mathrm{m}$ by using a tapered design for the microfluidic channel [Fig. $1 A$; the taper ranges from $300 \mu \mathrm{m}$ (inlet) to $100 \mu \mathrm{m}$ (outlet)] (Fig. $2 B)$. We increased the contrast in the refractive index $\left(\Delta n_{\mathrm{d}}>\right.$ $0.002)$ and the diameter of the core to $>10 \mu \mathrm{m}$ by increasing the rates of flow of both fluids $(\geq 5 \mu \mathrm{l} / \mathrm{min}(\tau \leq 0.09 \mathrm{~s})$ for the core and $\geq 20 \mu \mathrm{l} / \mathrm{min}$ ( $\tau \leq 0.06 \mathrm{~s}$ ) for the cladding) (Fig. $2 C$ and $D$ ); we expect multimode guiding for these dimensions and contrasts of the refractive indices (26).

In Fig. $2 B-D$, the beam is guided along the bottom of the channel. This position was caused by the difference in density between the core fluid $\left(\rho_{\text {aq. }} \mathrm{CaCl}_{2}=1.39 \mathrm{~g} / \mathrm{cm}^{3}\right)$ and the cladding fluid $\left(\rho_{\mathrm{H}_{2} \mathrm{O}}=1 \mathrm{~g} / \mathrm{cm}^{3}\right)$. The use of isodense fluids can minimize the influence of gravity on the system.

We measured the full width at half maximum of the output light from a 780-nm laser diode coupled into cores of different sizes (Fig. $2 E$ ). The dotted line in Fig. $2 D$ corresponds to diffraction-limited, single-mode operation reached with a core diameter of $8 \mu \mathrm{m}(27)$; we estimate that the large cores in Fig. $2 C$ and $D$ guide $\approx 5$ and $\approx 40$ modes, respectively (26). These data establish that the rates of flow of the core and cladding fluids dictate the number of modes of light carried by the liq/liq waveguides.

Optical Switch. We fabricated a microfluidic system that formed a liq/liq waveguide and then branched into three separate channels (Fig. 3). The relative rates of flow of the cladding fluids determined the path of the core fluid and thus the path of the light. Large differences $(>50 \%)$ in the rates of flow of the initial cladding streams caused deformation of the core stream (i.e., bowing and narrowing of the stream at the junction point) and thus loss of light. We added a separate set of inlets to this channel 
A
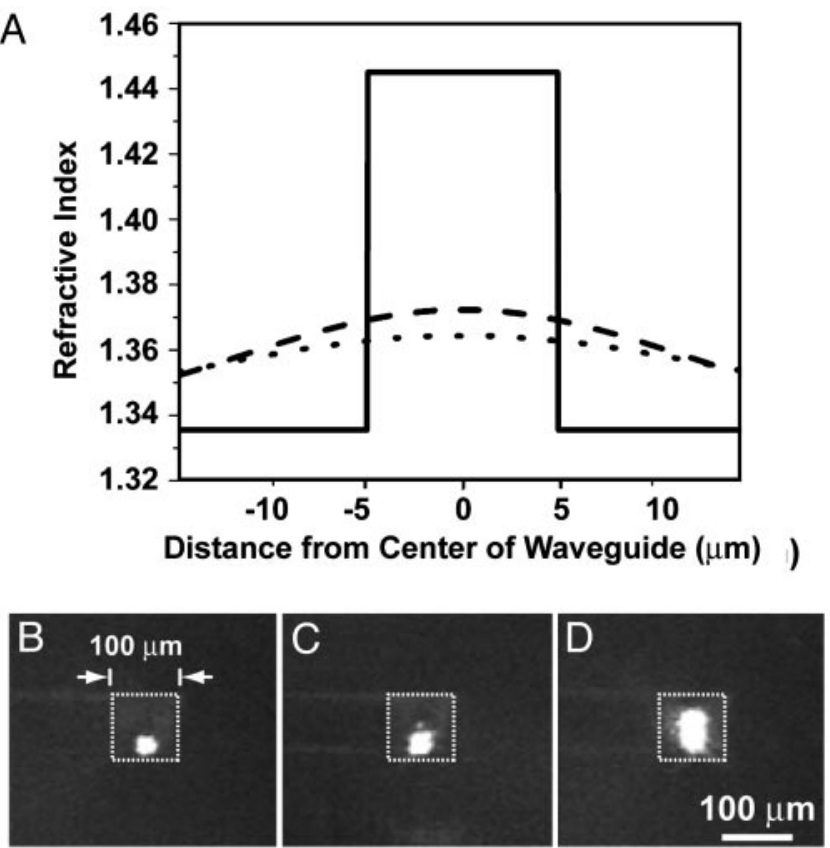

$10(0.135)$ core 2.5 (0.12) clad
$20(0.063)$ core $6.5(0.06)$ clad
$50(0.015)$ core 50 (0.022) clad
$\mathrm{E}$

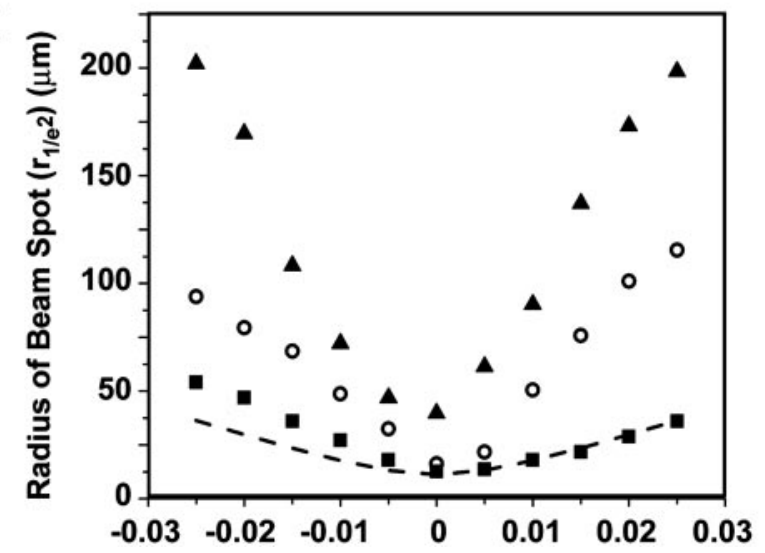

Fig. 2. Single-mode and multimode waveguiding. (A) Plot of the calculated refractive index as a function of distance from the center of the liq/liq waveguide for three positions along a waveguide of $5 \mathrm{~mm}$ in length [i.e., the beginning (solid line), the middle (dashed line), and the end (dotted line)] for the rates of flow (residence times) and channel dimensions in $B$. ( $B-D)$ Optical micrographs of the cross section of the outlet of the microfluidic channel viewed through the transparent window. The dashed box shows the location of the cross section of the microfluidic channel. The rates of flow $(\mu \mathrm{l} / \mathrm{min})$ (and the residence time in seconds) of the core and the cladding (clad) are listed. The guided light was from a fiber-coupled laser with $\lambda=780 \mathrm{~nm}$. (E) Plot of the radius $\left(r_{1 / \mathrm{e}^{2}}\right)$ of the beam spot as a function of distance (along the axis of diffraction) away from the location of the minimum spot size (as imaged through a microscope objective). The radius of the spot was measured only in the horizontal plane. The squares correspond to the flow rates in $B$. The open circles correspond to the flow rates in $C$. The triangles correspond to the flow rates in $D$. The dotted line is the theoretical prediction of the diffraction (beam spreading) of a single-mode beam with a minimum beam waist of $8.5 \mu \mathrm{m}$.

design downstream of the first set of inlets for the cladding fluids and used these independently controlled inlets to decrease the width of the core stream and to "push" the flow of the core fluid to one of the three outlets without generating sources of optical loss. This device required $\approx 2 \mathrm{~s}$ to switch from one output to another.

A
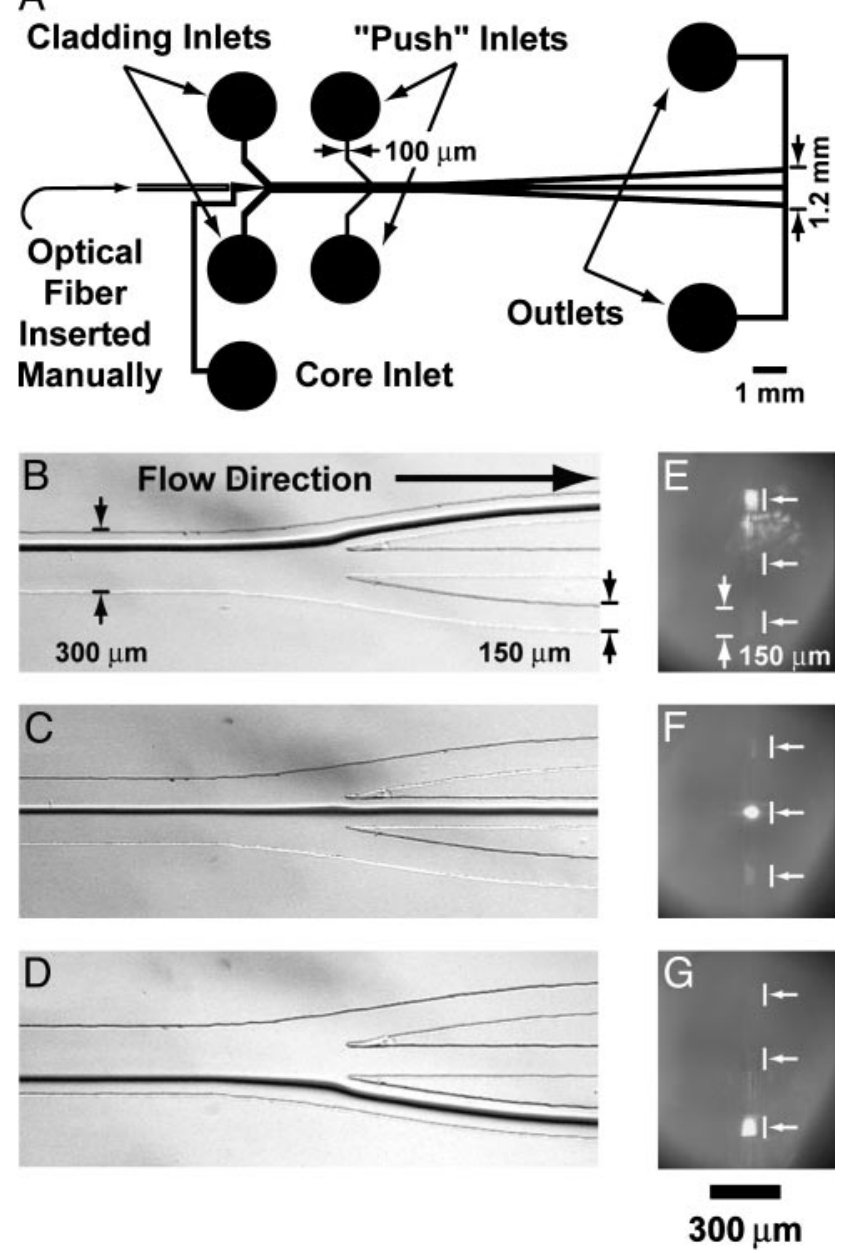

Fig. 3. Optical switch. (A) Schematic diagram of the microchannel used for the optical switch. $(B-D)$ Optical micrograph of the top view of the microfluidic channels. The core fluid (dyed) makes it easily imaged; the dye is omitted in use. $(E-G)$ Optical micrograph of the light that exits the liq/liq waveguides at the end of the channel system. The white arrows and lines represent the location of the ends of the branches of the microfluidic channel. The core fluid flowed through the center channel when the rates of flow of the cladding streams and of the push streams were approximately equal $(\approx 100 \mu \mathrm{l} / \mathrm{min})$; it moved to a side channel when the rates of flow were different (e.g., one cladding stream at $50 \mu \mathrm{l} / \mathrm{min}$ and the other at $125 \mu \mathrm{l} / \mathrm{min}$ and one push stream at $5 \mu \mathrm{l} / \mathrm{min}$ and the other at $175 \mu \mathrm{l} / \mathrm{min}$ ). The core fluid kinks slightly at the beginning of the branches in $B$ and $D$. We did not observe significant optical loss at this kink because its radius of curvature is greater than the critical radius $(\approx 1 \mathrm{~mm})$.

Evanescent Coupler. The design for a microfluidic-based evanescent coupler includes two liq/liq waveguides that share an inner cladding stream (Fig. $4 A$ and $B$ ). Light from an optical fiber is introduced into one of the liq/liq waveguides; we refer to this waveguide as the illuminated waveguide (IG). The width of the inner cladding stream can be adjusted by manipulating the rate of flow of the liquid; when this inner stream is sufficiently thin (or the value of refractive index contrast is sufficiently low), the evanescent fields of the two cores overlap and light transfers from the IG to the evanescently coupled waveguide [the coupled waveguide (CG)]. We used a microfluidic channel design with a narrow section (50 $\mu \mathrm{m}$ wide; Fig. $4 C)$ to obtain an inner cladding stream of the appropriate width $(<2 \mu \mathrm{m})$ to observe efficient coupling. We expanded the channel downstream from the coupling region to separate the IG and CG liq/liq waveguides and to simplify the character- 


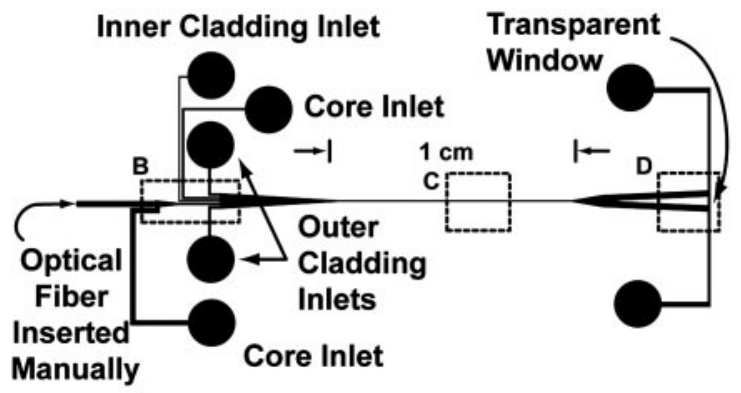

B
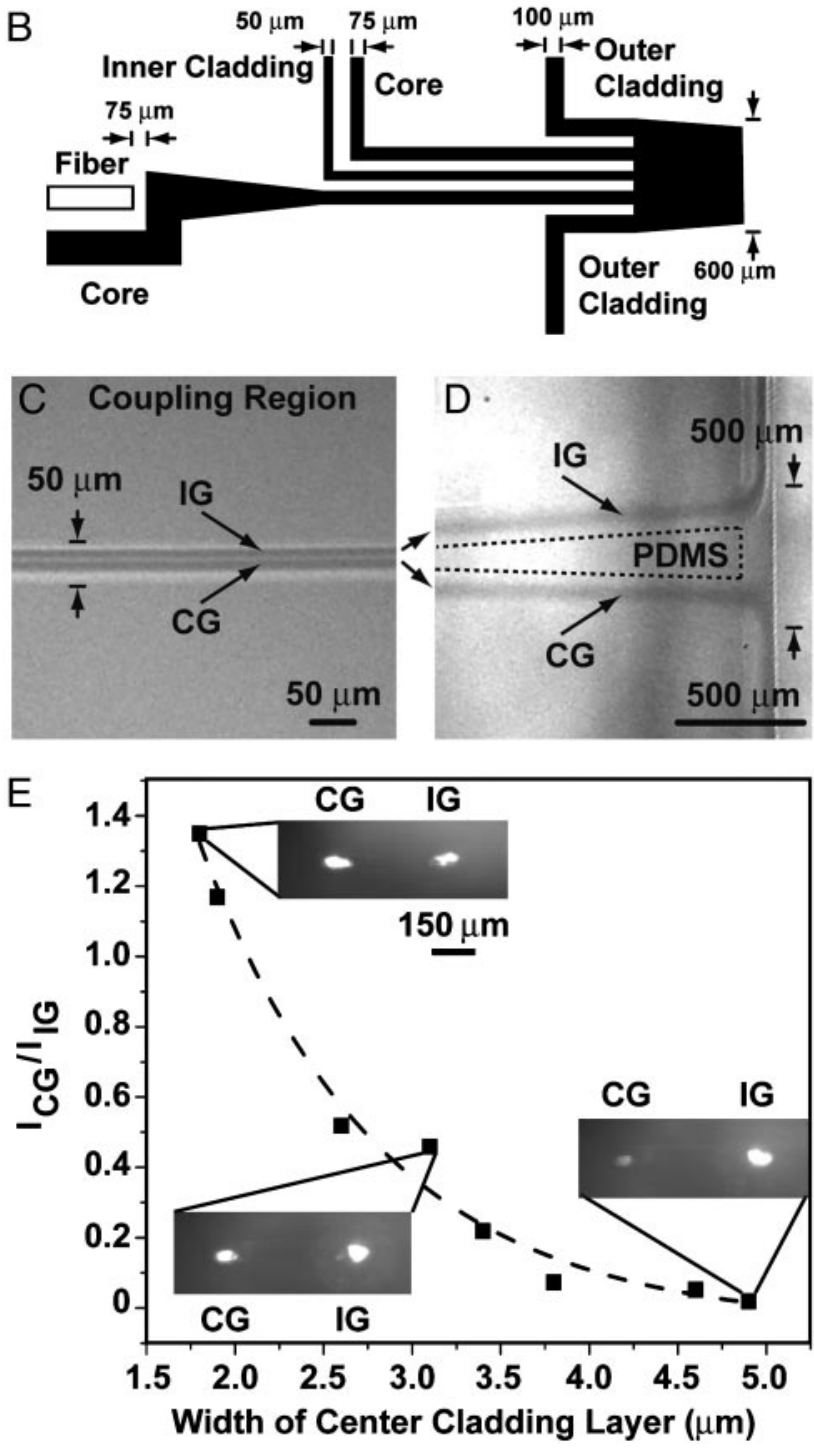

Fig. 4. Evanescent coupler. (A) Schematic diagram of the microfluidic channel for the evanescent coupler. $(B)$ Schematic diagram of the input region of the microfluidic channel. (C) Optical micrograph of the coupling region. (D) Optical micrograph of the end of the channel system where the light escaped and was imaged. The core fluid in $C$ and $D$ was dyed to make its visualization easy. (E) Plot of the ratio of the intensity of the light emitted from the coupled guide $\left(\mathrm{I}_{\mathrm{CG}}\right)$ and the illuminated guide $\left(\mathrm{I}_{\mathrm{IG}}\right)$. (Insets) Shown are optical micrographs of the cross section of the output of the microfluidic channels viewed through the transparent window.

ization of the guided light from each waveguide (Fig. 4D). Fig. $4 E$ shows ratios of the intensities of the light emitted from the end of the channel from each liq/liq waveguide as a function of the width of the inner cladding stream. It was difficult to maintain an inner cladding stream with a width of $<1.5 \mu \mathrm{m}$ with the microfluidic system used here; thus, we did not observe complete coupling of all of the light from the IG into the $\mathrm{CG}$.

We modeled the behavior of this device by using the program FREEBPM (www.freebpm.com). The refractive index contrast must be $\approx 0.01$ for this device to work at the distances between liquid cores that were observed. The operation of this device relies on the fact that diffusion of $\mathrm{CaCl}_{2}$ from the core to the cladding lowers the refractive index contrast in the coupling region. Simple estimates allow tuning of the rates of flow to achieve the desired refractive index contrast and thus the extent of coupling. The controlled use of diffusion of ions or dyes to adjust the refractive index contrast between flowing streams is unique to liq/liq optical systems.

\section{Discussion}

All-fluidic optical systems have three potentially useful differences from solid-state waveguides. (i) With liq/liq waveguide systems, it is easy to obtain small $(<10 \mu \mathrm{m})$ single-mode waveguides by using pressure-driven flow in large $(>100 \mu \mathrm{m})$ and easily fabricated channels. Solid-state devices require the use of high-resolution lithographic tools (laser or electron beam writers) to generate features with the lateral dimensions necessary for single-mode waveguiding; the microfluidic channels were written by using a high-resolution printer (28). (ii) The fluid flows at low $\mathrm{Re}$ generate an intrinsically optically smooth interface between the liquid core and liquid cladding. (iii) It is possible to change the properties of the liquid core and liquid cladding to alter the properties (e.g., the geometry) of the waveguide.

The disadvantages of the liq/liq systems include $(i)$ the constant supply of fluids necessary for both the cladding and the guiding streams (a supply of $250 \mathrm{ml}$ is necessary to run one stream at $175 \mu \mathrm{l} / \mathrm{min}$ for $24 \mathrm{~h}$ ); (ii) the mechanical stability is lower than solid-state waveguides; (iii) the inability (using water and PDMS ) to guide light of the wavelengths $(\lambda=1,300-1,600$ $\mathrm{nm}$ ) used in telecommunications applications because of large absorptive losses in both the fluid streams and in the PDMS; (iv) the response time of the pressure-based changes in the geometry of the guides (i.e., speed of optical switching; $\approx 0.1 \mathrm{~Hz}$ ) is much slower than switching in conventional planar waveguides $(\approx 1-$ $100 \mathrm{GHz}$ ). Many applications of waveguides in optical sensing, fluorescence microscopy, and bioassays often do not require high-speed switching.

The performance of the liq/liq waveguides can, in principle, be optimized continuously by using feedback to change in real time the rates of flows of the liquid components. The size of the beam is important for spatially sensitive measurements, such as illumination of a single cell in a microfluidic channel. The mode structure of the beam is important to achieve optimum signalto-noise ratios. Additional optics (e.g., lenses or pinholes), therefore, are necessary to shape the beam within solid-state waveguides. We believe that optical devices based on this methodology may be useful for on-chip analysis devices that use optical excitation and detection because it is relatively simple to change the characteristic (i.e., intensity, shape, and location) of the beam spot.

This system provides a strategy for the fabrication of tunable optical devices that have a level of reconfigurability that is difficult, if not impossible, to obtain with solid-state waveguides.

We thank J. Christopher Love, Dmitri Vezenov, and James Carey for helpful discussions. This work was supported by the Center for Optofluidic Integration at the Defense Advanced Research Projects Agency and a postdoctoral fellowship from the Foundation for Polish Science (to P.G.). 
1. Stroock, A. D., Dertinger, S. K. W., Ajdari, A., Mezic, I., Stone, H. A. \& Whitesides, G. M. (2002) Science 295, 647-651.

2. Ma, H., Jen, A. K. Y. \& Dalton, L. R. (2002) Adv. Mater. 14, 1339-1365.

3. Xia, Y. \& Whitesides, G. M. (1998) Angew. Chem. Int. Ed. Engl. 37, 550-575.

4. Xia, Y. \& Whitesides, G. M. (1998) Annu. Rev. Mater. Sci. 28, 153-184.

5. McDonald, J. C., Duffy, D. C., Anderson, J. R., Chiu, D. T., Wu, H., Schueller, O. J. A. \& Whitesides, G. M. (2000) Electrophoresis 21, 27-40.

6. McDonald, J. C. \& Whitesides, G. M. (2002) Acc. Chem. Res. 35, 491-499.

7. Schueller, O. J. A., Zhao, X.-M., Whitesides, G. M., Smith, S. P. \& Prentiss, M. (1999) Adv. Mater. 11, 37-41.

8. Manor, R., Datta, A., Ahmad, I., Holtz, M., Gangopadhyay, S. \& Dallas, T. (2003) IEEE Sens. J. 3, 687-692.

9. Datta, A., Eom, I.-Y., Dhar, A., Kuban, P., Manor, R., Ahmad, I., Gangopadhyay, S., Dallas, T., Holtz, M., Temkin, H. \& Dasgupta, P. K. (2003) IEEE Sens. J. 3, 788-795.

10. Dress, P. \& Franke, H. (1996) Appl. Phys. B: Lasers Opt. 63, 12-19.

11. Dijkstra, R. J., Slooten, C. J., Stortelder, A., Buijs, J. B., Ariese, F., Brinkman, U. A. T. \& Gooijer, C. (2001) J. Chromatogr. 918, 25-36.

12. Hanning, A., Westberg, J. \& Roeraade, J. (2000) Electrophoresis 21, 3290-3304

13. Wang, S.-L., Huang, X.-J., Fang, Z.-L. \& Dasgupta, P. K. (2001) Anal. Chem. 73, 4545-4549.

14. Liu, Z. \& Pawliszyn, J. (2003) Anal. Chem. 75, 4887-4894.
15. Olivares, J. A., Stark, P. C. \& Jackson, P. (2002) Anal. Chem. 74, 2008-2013.

16. Dress, P. \& Franke, H. (1997) Rev. Sci. Instrum. 68, 2167-2171.

17. Domachuk, P., Nguyen, H. C., Eggleton, B. J., Straub, M. \& Gu, M. (2004) Appl. Phys. Lett. 84, 1838-1840.

18. Mach, P., Dolinski, M., Baldwin, K. W., Rogers, J. A., Kerbage, C., Windeler, R. S. \& Eggleton, B. J. (2002) Appl. Phys. Lett. 80, 4294-4296.

19. Kerbage, C. \& Eggleton, B. J. (2003) Appl. Phys. Lett. 82, 1338-1340.

20. Cattaneo, F., Mach, P., Hsieh, J., Krupenkin, T., Yang, S. \& Rogers, J. A. (2003) Mater. Res. Soc. Symp. Proc. 741, 9-13.

21. Cattaneo, F., Baldwin, K. W., Yang, S., Krupenkine, T., Ramachandran, S. \& Rogers, J. A. (2003) J. Microelectromech. Syst. 12, 907-912.

22. Takiguchi, H., Odake, T., Ozaki, M., Umemura, T. \& Tsunoda, K.-I. (2003) Appl. Spectrosc. 57, 1039-1041.

23. Lee, J. N., Park, C. \& Whitesides, G. M. (2003) Anal. Chem. 75, 6544-6554.

24. Tamir, T. (1998) Guided-Wave Optoelectronics (Springer, New York).

25. Ismagilov, R. F., Stroock, A. D., Kenis, P. J. A., Stone, H. A. \& Whitesides, G. M. (2000) Appl. Phys. Lett. 76, 2376-2378.

26. Yariv, A. (1996) Optical Electronics in Modern Communications (Oxford Univ. Press, New York).

27. Siegman, A. E. (1986) Lasers (University Science Books, Sausalito, CA).

28. Linder, V., Wu, H., Jiang, X. \& Whitesides, G. M. (2003) Anal. Chem. 75, 2522-2527. 